\title{
Erratum to: Assessing environmental risks for high intensity agriculture using the material flow analysis method - a case study of the Dongting Lake basin in South Central China
}

\author{
Guanyi Yin • Liming Liu • Chengcheng Yuan
}

Published online: 24 November 2015

(C) Springer International Publishing Switzerland 2015

Erratum to: EnvironMonit Assess (July 2015) 187, Issue 7:4696

DOI 10.1007/s10661-015-4696-y

The original article contained an error.

The first author affiliation should have been China Agricultural University not Agricultural University.

The corrected affiliation is shown below.

The online version of the original article can be found at http://dx. doi.org/10.1007/s10661-015-4696-y.

G. Yin · L. Liu - C. Yuan

Department of Land Resources Management, College of Resource and Environmental Sciences, China Agricultural University,

Beijing 100193, China

L. Liu $(\bowtie)$

Department of Land Resources Management, China Agricultural University, No.2 Yuanmingyuan West Road, Haidian District,

Beijing 100193, China

e-mail: liulm@cau.edu.cn 